\title{
Developing Low-Cost Intelligent Wireless Sensor Networks for Aquatic Environments
}

\author{
Jarrod Trevathan*, Ian Atkinson*, Wayne Read*, Nigel Bajema*, Yong Jin Lee*, Adam Scarr* and Ron Johnstone ${ }^{\dagger}$ \\ *eResearch Centre - James Cook University, Townsville, Australia \\ ${ }^{\dagger}$ School of Geography, Planning and Environmental Management - University of Queensland, Brisbane, Australia
}

\begin{abstract}
Aquatic environments are extremely difficult for developing, deploying, and maintaining wireless sensor networks. Networks deployed in aquatic settings face multiple challenges, such as marine fowling of equipment, limited power supply, communications difficulties, and restricted accessibility for maintaining and updating sensor nodes. The SEMAT project is an initiative to create "smart", low-cost, heterogeneous wireless sensor networks, tailored to alleviating the aforementioned constraints. Networks can be instantly deployable with minimal setup overheads and can utilise equipment from multiple vendors. This paper presents our experiences with developing the initial technologies to establish SEMAT for field tests. We present the design methodology and challenges faced for creating a marinebased heterogeneous wireless sensor network platform. The result is a low cost solution, with sufficient accuracy for undertaking a study into the factors contributing to Lyngbya algae blooms in Deception Bay, Queensland. The platform builds a case for the merits of the final SEMAT system, as ultimately many of the software and basic hardware challenges for future aquatic deployments have been overcome. This is significant as it allows researchers to focus on the area under study, rather than the specifics of setting up and managing the network.
\end{abstract}

\section{INTRODUCTION}

Wireless Sensor Networks (WSNs) allow for multiple sensor nodes to collect information on phenomena of interest which is wirelessly communicated back to an end-user. WSNs are more versatile than their traditional wired counterparts, and can be deployed over larger areas which leads to more potential applications for their use. Some of the domains where WSNs are being applied include health monitoring, environmental monitoring and security. However, the physical environment raises significant challenges which often makes deployment very difficult.

Marine environments are particularly harsh for deploying WSNs. Networks are typically dispatched in remote locations with equipment having to be stationed on the sea floor, a river bed, or on a turbulent surface where wave action or river current may dislodge equipment and/or hamper communications. Some specific problems for aquatic WSNs include marine fowling from moss, algae, or animals that damage or prevent sensor nodes from undertaking their desired functions. Furthermore, there are issues with initially deploying such systems underwater and maintaining/updating equipment and software. This may range from using divers to expensive submersible vehicles. The next constraint is on power usage and the expense involved with repowering devices that have exhausted their power supplies. Furthermore, the marine environment presents unique difficulties for data transmission and communications between sensor nodes and gateways. Finally, sensor packaging must be water tight and resilient to the conditions of turbidity, salinity and water pressure.

The Smart Environmental Measurements and Analysis Technologies (SEMAT) system plans to solve many of these problems by offering an end package of "intelligent" sensors and analysis tools that can be instantly deployed in any aquatic environment [4]. SEMAT has recognised the need for inexpensive WSNs that can be deployed without having to rely on a large team of experts including engineers, computer scientists, and marine biologists [7]. SEMAT aims towards having nodes that are "plug and play", in that new nodes can be added to the system which are instantly recognisable without having to reconfigure the system. Furthermore, as nodes can potentially be deployed in the deepest regions of the ocean, SEMAT will allow for wirelessly powered nodes, so that human intervention is never required to charge them. Finally, sensors are intelligent in that they contain algorithms that allow groups of sensors to make autonomous decisions independent of the end user in the case that certain predefined conditions are met [5].

This paper discusses the initial methodology behind creating the SEMAT system. We present a basic WSN for gathering data from a marine environment for SEMAT's first field tests. We fully describe the software and hardware used and the design methodology behind the decisions made. The result is a low cost solution, with sufficient accuracy for undertaking a study into the factors contributing to Lyngbya algae blooms in Deception Bay, Queensland. The platform builds a case for the merits of the final SEMAT system, as ultimately many of the software and basic hardware challenges for future aquatic deployments have been overcome. This is significant as it allows researchers to focus on the area under study, rather than the specifics of setting up and managing the network.

This paper is organised as follows: Section II describes the SEMAT project's goals and expected outcomes. Section III discusses the SEMAT test development site, the phenomenon under study, and the deployment model. Section IV describes the specifics and rationale of the hardware, software, communications, sensors and power/data management. Section V provides some concluding remarks and discusses the future direction for the SEMAT project. 


\section{SEMAT}

This section briefly describes the SEMAT project. The specific goals for SEMAT include:

1) Underwater wireless communications As aquatic environments are remote/vast, it is not economically viable or practical to have sensors wired together. Often in such an environment the positioning of the cables represents a significant practical problem. The cable itself is also vulnerable to breakage or degradation over time.

2) Short-range wireless and power transmission Interconnecting cables for data communications and/or power insitu is complex in a marine environment. SEMAT aims to develop technologies whereby neighbouring cables can be connected underwater and inductive methods used to transfer power and data between nodes.

3) Plug and Play A major problem facing the deployment of WSNs is the disparate technologies used as equipment must be combined from different manufacturers. Even the simple case of adding a new type of sensor usually involves reconfiguring the entire system so that the end user can view the sensors output. SEMAT will use SAL (see Section IV-B1) to allow new equipment to be added such that it is instantly recognized and configured for use. Making a WSN plug and play removes much of the technical overhead for managing the network.

4) Minimal deployment expertise SEMAT will offer end users a complete package. The end user will only need to choose what sensors they require and SEMAT will autoconfigure the necessary parameters. Essentially once deployed, the user could take a laptop down to the beach (for example) and can begin to view the sensed data.

5) Near real-time analysis tools SEMAT will provide software tools that allow data to be streamed in near realtime from sensors. Users will have the ability to buffer large amounts of data and sift through it using the RBNB Data Turbine [6]. Data collected is put into a format that is recognized by standards bodies (i.e., Sensor Web Enablement [2]) and therefore can be imported into sophisticated data modelling and visualization tools.

6) Intelligent sensors Sensor nodes will have a level of intelligence in that they have two way communications with each other. This will allow sensors to have a degree of autonomy from the end user such that if there is a sudden change in a condition which affects the phenomena under study, then sensor nodes can communicate with each other to change their parameters to better study the changes in the environment. For example, if one set of sensors detects that significant rainfall is occurring, it might communicate with the salinity sensor to increase its sensing rate from daily to hourly. There are almost limitless uses for such intelligence.

SEMAT is an ambitious and large project which draws on expertise from multiple disciplines including marine biology, engineering, mathematics, and computer science. There are several parties involved with the development of SEMAT:

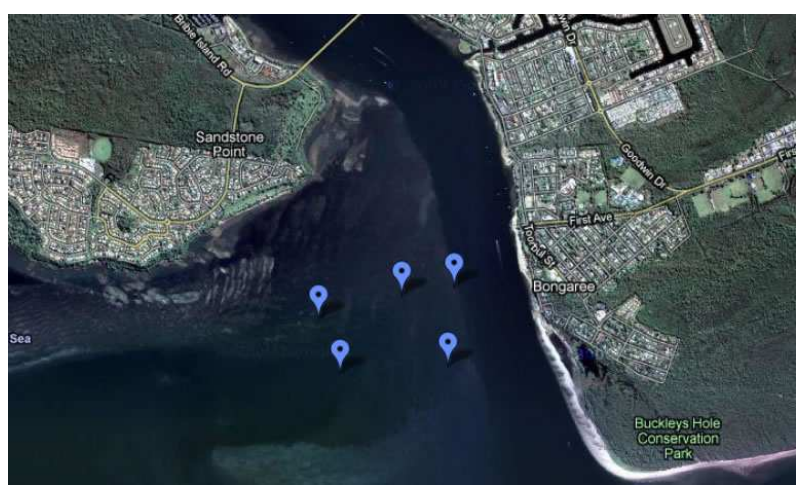

Fig. 1. Location of sensor stations in the Deception Bay study area

University of Queensland (UQ), DHI, James Cook University (JCU) and the Torino Foundation.

DHI and UQ are responsible for the marine science and high level modelling of marine phenomena. For the purposes of SEMAT, this study is related to the prevalence of Lyngbya algae blooms at Deception Bay in Queensland Australia. DHI have developed sophisticated modelling software that is being used for the initial studies SEMAT will be conducting.

The Torino Foundation is a prominent Italian company who specialise in sensor technology. They are tasked with designing purpose built intelligent sensors for the SEMAT system. The initial sensors under development will be geared towards the studies being undertaken by DHI and UQ.

The Information Technology and Electrical Engineering school at UQ is designing the wireless power transfer and communications systems. They are working in conjunction with the Torino Foundation to decide on the electrical standards for the power transfer. UQ is also working in conjunction with JCU on the communications protocols that will be used between sensor nodes and how the information will be relayed back to the gateway devices.

The eResearch Centre at JCU is responsible for providing the middleware and end user interface for the data acquisition, storage and presentation from the SEMAT system. JCU is tasked with making the system plug and play and allowing for near real-time analysis of the collected data. This paper will focus on JCU's role with setting up the initial SEMAT system for a trial in the SEMAT development environment.

\section{Development Site And Deployment Model}

This section describes the deployment site and phenomenon under study for the SEMAT prototype system.

Lyngbya Algae is a particularly virulent form of algae that attaches itself to seagrass. The algae absorbs all of the nutrients from the sea floor and surrounding areas and essentially kills off the sea grass. Once the algae has finished growing, it breaks off and typically washes up on the beach/shore line. It leaves scaring along the sea bed where it was present and it usually prevents future seagrass from growing.

While it is uncertain what causes the algae, it is suspected that it forms mainly in warm conditions, shallow and calm water, and human activity (possibly in the form of nutrient run off) may affect its life cycle. The initial SEMAT case 


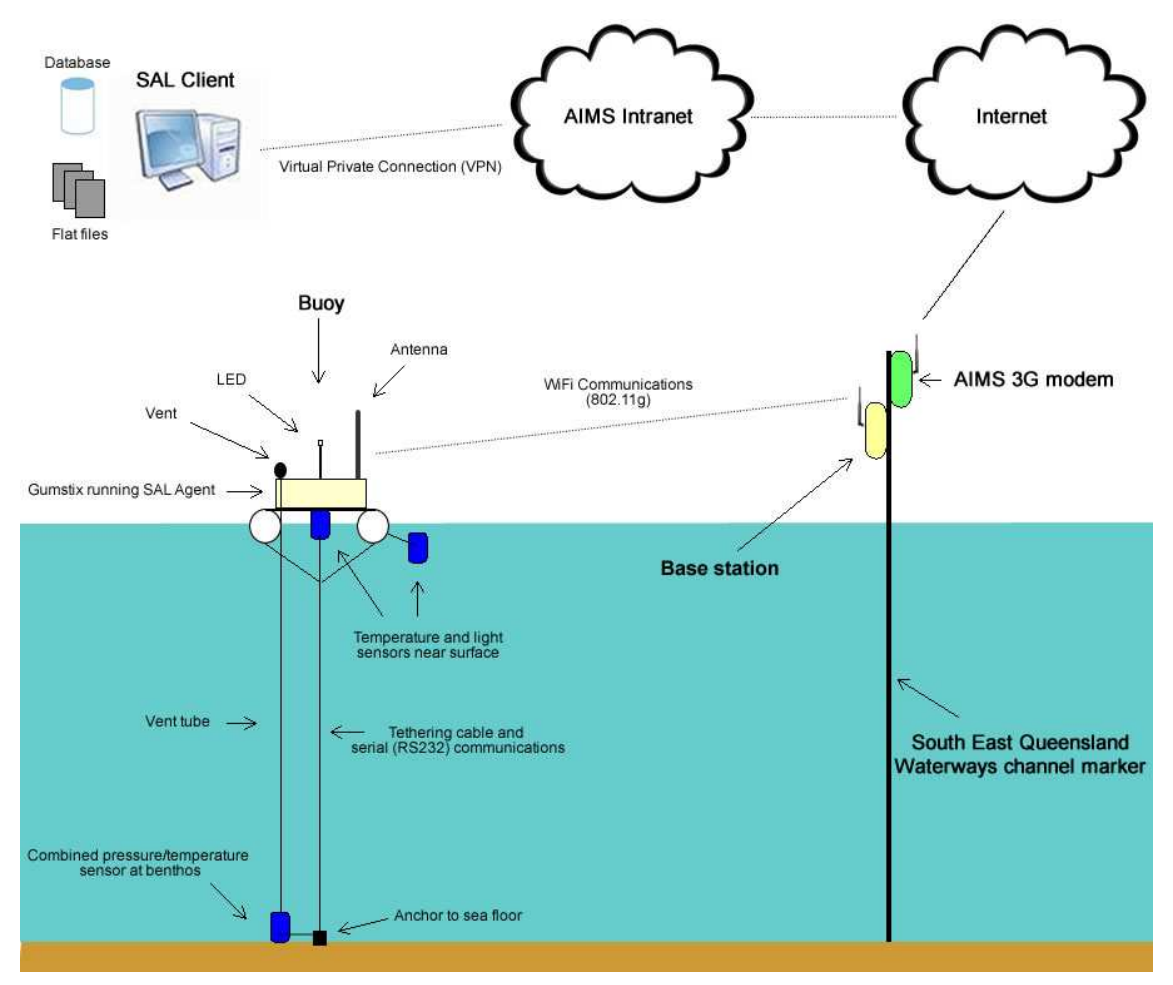

Fig. 2. The basic setup of a sensor station for the core Deception Bay trial

study is to examine the influences contributing to the spread of Lyngbya algae. Ideally the result will be to suggest an environmental management plan to control its spread in aquatic environments.

In recent years, Deception Bay in Queensland Australia has experienced significant Lyngbya algae blooms. The bay is largely protected from the greater ocean and therefore doesn't contain any significant wave action. It is shallow with high tide only raising the water depth to approximately three metres at its peak. Deception Bay is situated between the mainland and the southern region of Bribie island, therefore it is heavily influenced by human activity.

As the initial application for SEMAT is for monitoring aquatic environments for marine science studies, UQ and DHI selected Deception Bay as the premiere site for testing the initial SEMAT system. There were several factors influencing this decision: 1) Proximity to Brisbane; 2) Prevalence of algal blooms; 3) Subtropical environment; 4) Shallow water; and 5) Calm conditions. Figure 1 shows the locations where core measurements will be taken for these factors within the bay.

There are three aspects to the SEMAT Deception Bay deployment. The main or core deployment is designed to emulate SEMAT's goals as closely as possible, but at this stage lacks the underwater wireless communications and powering component. There will also be a shadow deployment where straight logging devices without real-time communications will be placed alongside the core deployment. This will be used for backup and calibration purposes. Finally there will be a satellite deployment of numerous other forms of sensing devices at various other locations around the bay. However, none of these will form part of the SEMAT WSN.
The core deployment is divided into three types of sensor stations depending on location, the number of sensors and the types of sensors used. These sensor station types are referred to as: Primary, Secondary and Tertiary. Out of the five core sensor stations, there is one primary station, two secondary, and two designated as tertiary.

\section{Deployment SPECIFICS}

Figure 2 illustrates how the sensor stations will be configured (from a high level perspective). Each sensor station consists of a buoy that is tethered to the sea floor via a cable. Figure 3 illustrates the internal components of each sensor station from the core deployment. This section will describe each of the characteristics in turn below.

\section{A. Sensors}

The Lyngbya algae study requires several types of characteristics to be measured. The initial deployment (i.e., the combined core, shadow and satellite) aims to collect the following information: dissolved oxygen, light, pressure, salinity, temperature and turbidity. The core deployment will only be concerned with light (photosynthetic irradiance), temperature and pressure. The shadow and satellite deployments will gather data on the other aspects. (Note that a pressure sensor requires a nylon vent tube connecting it to a vent at the surface.)

Dataflow Systems is a New Zealand-based company that supplies a range of terrestrial and aquatic logging devices. These are referred to as Odyssey loggers. A logger is designed to be a standalone unit complete with its own casing, independent power supply and onboard memory. A user initially sets up the logger by connecting it to a computer via a 


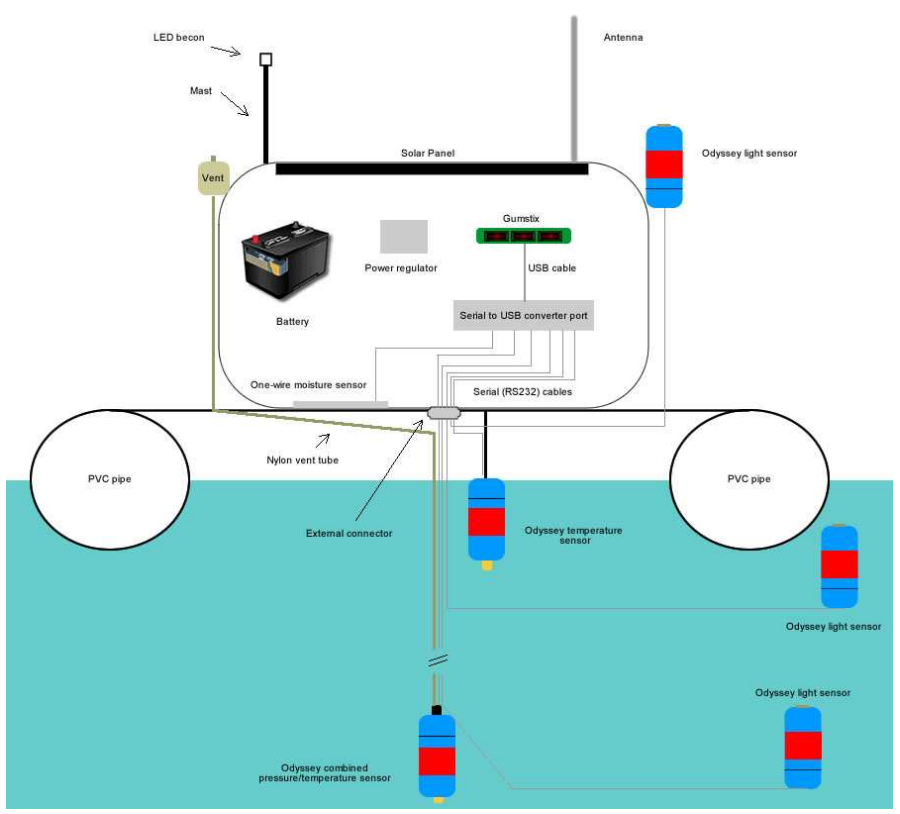

Fig. 3. Internal components of a sensor station

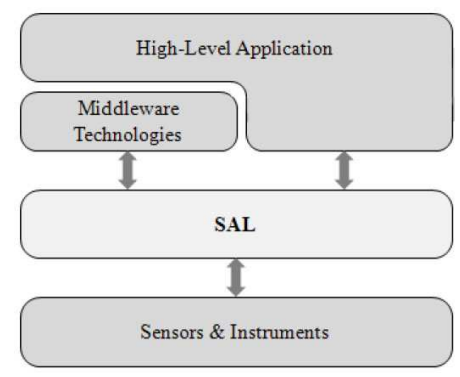

Fig. 4. The SAL software model. SAL sits between the lower middleware and the hardware for the sensors and instruments. It facilitates a transparent interface for communication between the high-level application programs and the sensor hardware.

serial/USB cable. The user is required to be running Odyssey's proprietary software. Once the logger has been started, the cable is disconnected from the device, the cap screwed on and then the device is deployed. At the end of the deployment, the device is retrieved, the cable attached, and then the data is downloaded to a computer. Depending on the sensor type, the sensing interval can be set between 10 seconds and eight hours. Theoretically, Odyssey loggers can be deployed for up to 18-24 months depending on the sensing interval.

While the regular method of use will suffice for the shadow and satellite SEMAT deployments, the core deployment will involve a more interactive approach. Here the loggers will be permanently attached to a computer and will be able to be started and stropped in real-time during deployment (without having to be retrieved). The data will be downloaded and transmitted back to the end user.

\section{B. Software}

1) Sensor Abstraction Layer (SAL): Sensor networks (SNs) are becoming increasingly essential as sensors are introduced into more areas of life. However, lack of standards makes it difficult to integrate heterogeneous sensors in a single $\mathrm{SN}$.

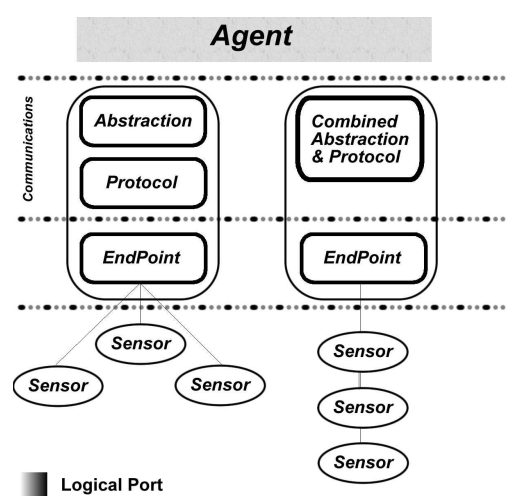

Fig. 5. SAL components and software layers

Therefore it is important to have middleware which is able to manage all types of sensors. This middleware is often implemented specifically for one SN, which makes it hardware dependent. Changes in the network, such as adding a new sensor, leads to manipulating the middleware code.

SAL is a middleware integration platform which provides a plug-in-based model where support for new types of sensors can be loaded to the running system via plug-in. The system automatically detects and configures new sensors, if permitted by the hardware and operating system (OS). It provides a unified interface to all sensors by abstracting the sensor specific features. This simplifies access to a $\mathrm{SN}$ and the management/control of its sensors [3]. SAL can be seen as a low-level software layer as it bridges a network of sensors with high-level applications or further middleware technologies.

SAL consists of two components, the SAL client and the SAL agent. The SAL client represents an interface for SAL to either the user via a user interface or to other applications. It implements the SAL agent Application Programming Interface (API) in order to provide the SAL agent's functionality. The API is grouped into the following categories:

- Sensor Management - Methods to manage the pool of sensors and including operations for enumerating, adding and removing sensors.

- Sensor Control - Methods to report on a sensor's capabilities and control the streaming of the data.

- Platform Configuration - Methods to adjust the platform, e.g., add support for a new sensor type.

Each category uses a different markup language. The Sensor Management methods use SensorML [1], which describes a sensor's configuration. The methods in the category Sensor Control use CommandML. The CommandML documents contain a list of commands which are supported by a sensor. The last category, Platform Configuration, uses Platform Capabilities and Configuration Markup Language (PCML). PCML documents contain information on the platform configuration in order to support a certain type of sensor technology.

The SAL agent implements the various features of SAL. It runs on a platform which is connected to the sensors and therefore is regarded as a sensor gateway. The connection between the platform and the sensors can be either direct using platform specific input/output (I/O) ports like USB or 
indirect by using wireless technology. The SAL agent manages the sensors which are directly connected and are found by the agent and the indirectly connected sensors it has been told of. The SAL agent consists of three layers: Agent Layer, Communication Layer, and EndPoint Layer (see Figure 5).

The Agent Layer is responsible for the communication with the SAL client. It receives messages, parses and forwards them to the underlying Communication Layer and sends the response back to the client.

The Communication Layer provides methods for managing and controlling sensors. The managing methods are used to configure and set up hardware while the controlling methods translate a generic command into a sensor native command, which then can be transmitted to the sensor. The generic commands are provided in the SAL API. For translating the generic commands two sub-layers are used. The Abstraction Layer is an adapter layer where the generic commands are implemented. From here the sensor-specific methods are called, which are implemented in the Protocol Layer.

The EndPoint Layer is tightly coupled to the I/O ports available on the sensor gateway. It is responsible for transmitting native sensor commands produced by the Protocol sub-layer to the sensor, and data from the sensor is transmitted to the SAL agent. This layer's software code layer is normally included in the OS. SAL ensures it is available and configured correctly.

The sub-layers Abstraction and Protocol and the EndPoint Layer form a Logical Port. Each sensor is connected to a Logical Port which allows a specific client to control multiple agents. The logical port allows SAL to scale vertically in that an agent can control other agents in a hierarchical manner. The logical port treats an attached device as a data source, independent of whether it is a sensor or another agent. The ability to scale allows SNs of almost any topology or configuration.

2) Shadow Deployment: The shadow deployment uses the proprietary Odyssey software. This software is used to start up and shut down the loggers. It also provides functionality for calibration and also a basic charting facility for viewing the collected data. SAL also allows for files to be exported into Odyssey's software for charting.

\section{Hardware}

1) Sensor Stations: Each sensor station will use its own Gumstix Overo Air computer-on-module. These devices have a processor speed of $600 \mathrm{MHz}$ and provide $256 \mathrm{MB}$ of RAM. The Chestnut board is an additional circuit required by the Gumstix that supplies it with power and allows peripherals to be attached. A Serial to USB convertor port is also required as the Odyssey loggers only support serial communication.

2) Base Station: The base station is located on the Southeast Queensland Waterways channel marker. This is a 'dumb' node in that it does not contain any computing device (such as a gumstix). Instead it is purely a communications rely that interacts directly with the Australian Institute of Marine Science (AIMS) 3G modem.

3) Shadow and Satellite Deployments: The shadow deployment contains the same Odyssey loggers as the core

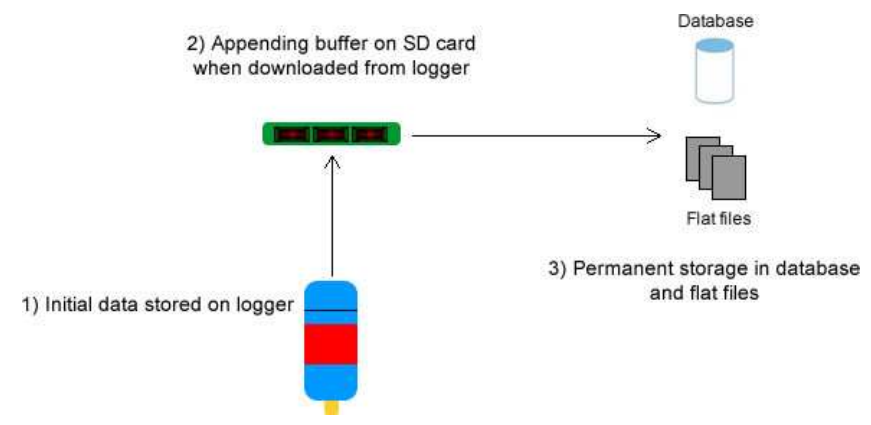

Fig. 6. Overview of the data backup and storage hierarchy

deployment. However, it doesnt require the computing and communications hardware. The satellite deployment has additional sensing devices.

\section{Communications}

The shadow and satellite deployments will require no permanent communications infrastructure. All loggers will have to be manually retrieved and the data downloaded.

For the core deployment, each Odyssey logger is physically connected to the Gumstix via serial RS232 cables. As the Gumstix only supports USB connections, a serial to USB port is required. Each serial cable (three in total for a sensor station) will connect directly to the port. The port will then convert the signals to USB, which then connects to the Gumstix.

Wireless (WiFi $802.11 \mathrm{~g} / \mathrm{n}$ ) communications are used to relay the information back to the communications gateway located on the floating platform. In the best-case scenario this has a throughput of $150 \mathrm{Mbit} / \mathrm{s}$ using a $2.4 \mathrm{GHz}$ band.

A $3 \mathrm{G}$ modem relays this back via the Internet and the AIMS Intranet to an end user (also running SAL software). An end user running a SAL client can VPN into AIMS. The AIMS 3G modem will be positioned on the existing Queensland Waterways channel marker at Deception Bay.

\section{E. Data Backup and Storage}

There are three main tiers to storage and back up of the data: The logger (1st tier - transient); An SD card attached to the Gumstix (2nd tier - semi-permanent); The database and flat files (3rd tier - permanent)

Initially data is stored in the internal memory of the logger. Odyssey loggers contain $64 \mathrm{~KB}$ of memory (65,528 bytes). The temperature and light sensors for this deployment store 2 bytes per reading. The temperature/pressure sensor records 4 bytes per reading (i.e., 2 bytes for temperature and 2 bytes for pressure). The amount of memory is capable of storing 32,764 records. A scan interval of 15 minutes has 96 recordings each day. The total number of days is 341 days before the memory capacity will be used up. When the memory is full, the logger shuts down. Note that if the logger malfunctions before it is uploaded, then the data for that interval will be lost.

When the logger is shut down, the data is transferred to an $\mathrm{SD}$ card on the Gumstix. Once the logger is started up again, its memory is cleared. The SD card contains an appending buffer. Every time data is downloaded from a logger, a copy is retained on the SD card (until the deployment finishes). 


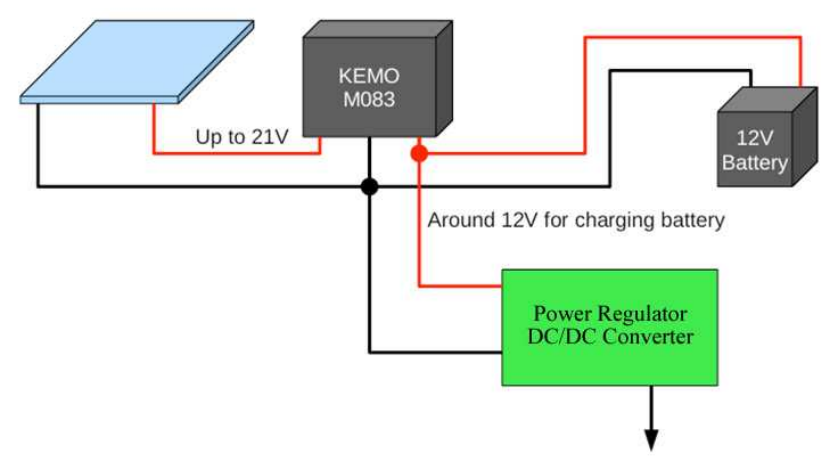

Regulated $5 \mathrm{~V}$ and $12 \mathrm{~V}$ out

Fig. 7. Overview of the power system

Assuming a duty cycle of two hours, 64 bytes of sensor readings plus overhead will be transmitted per sensor station. ( 8 bytes per reading x 815 minute readings over two hours.)

The final tier in the memory hierarchy is permanent storage in a database or flat files. This is handled by the SAL client. The database is implemented in MySQL and can be queried to retrieve specific data regarding the sensor readings. The flat files are straight text files where the sensed data is appended to existing records. The files can be imported into a spreadsheet or used as input to other external programs.

\section{F. Power Management}

1) Battery: Each Odyssey logger contains an independent power supply (two 3.6V Lithium cells $7.2 \mathrm{~V}$ in total). Loggers are independent of the main power supply for a sensor station. Battery life depends upon how frequently the user logs data. For a logging time of every 1-hour, batteries will last for more than 18 months under normal operating conditions.

Figure 7 gives an overview of the power system for each sensor station. Each station contains a $12 \mathrm{~V} 7 \mathrm{Ah}$ sealed lead acid battery to power the Gumstix and associated peripherals. Tests indicated that this battery has a lifetime of approximately 20 hours from a full charge with a load placed on it.

2) Solar Panels: Each sensor station will contain a solar panel to charge the battery during day light hours. The solar panel can generate up to $21 \mathrm{~V}$. A solar regulator (SunSaver SS$6 \mathrm{~L})$ will step this down to the battery voltage first. A power regulator then reduces the battery voltage down to the $5 \mathrm{~V}$ required for the Overo.

3) Duty Cycling: This a technique used to reduce power consumption by powering down (or hibernating) a sensor station in between sensing activities. The SAL software contains the functionality to start and stop the Odyssey loggers on command. When starting a logger, it configures it to take an independent reading at 15 minute intervals. The station then powers down until it is instructed to turn back on, or if a predetermined cycling schedule is implemented.

The system adheres to the following schedule for cycling. Here a cycle refers to powering up, stopping all loggers, downloading their data to the Gumstix, communicating it back to the end user via the base station, restarting all the loggers, and then powering down again. A station initially powers up at $10 \mathrm{am}$, transmit its data, and then powers down. It does this every two (2) hours through to $4 \mathrm{pm}$. It stays powered down all night until 10 am the next morning. The goal is to conserve battery life by only using the system during the day when the solar panels can compensate for the power consumption.

Note that this is a staggered schedule so that each sensor station gains exclusive access to the base station for the period it is active. That is, the first station will cycle on from $10 \mathrm{am}$ to $10: 15 \mathrm{am}$, the second from $10: 15$ to $10: 30$, etc.

\section{CONCLUSIONS}

This paper presents our experiences with developing the initial technologies to establish SEMAT for field tests. We present the design methodology and challenges faced for creating a marine-based heterogeneous wireless sensor network platform. The result is a low cost solution, with sufficient accuracy for undertaking a study into the factors contributing to Lyngbya algae blooms in Deception Bay, Queensland. The platform builds a case for the merits of the final SEMAT system, as ultimately many of the software and basic hardware challenges for future aquatic deployments have been overcome. This is significant as it allows researchers to focus on the area under study, rather than the specifics of setting up and managing the network. The work presented here also allows for the SEMAT project to continue to the next stage - development of wireless underwater power transfer and communications.

\section{ACKNOWLEDGMENT}

This work was supported in part by the Queensland Government National and International Research Alliances Program.

\section{REFERENCES}

[1] G. Aloisio, D. Conte, C. Elefante, G. P. Marra, G. Mastrantonio, and G. Quarta, "Globus monitoring and discovery service and SensorML for grid sensor networks", In Proceedings of the 15th IEEE International Workshops on Enabling Technologies: Infrastructure for Collaborative Enterprises, pp. 201-206, 2006.

[2] M. Botts, G. Percivall, C. Reed and J. Davidson: OGC Sensor Web Enablement: Overview and High Level Architecture. GSN 2006, LNCS 4540, pp. 175-190, 2008.

[3] G. Gigan, I. M. Atkinson, "Sensor Abstraction Layer: a unique software interface to effectively manage sensor networks", In Proceedings of the International Conference on Intelligent Sensors, Sensor Networks and Information Processing, pp. 479-484, 2007.

[4] R. Johnstone, D. Caputo, U. Cella, A. Gandelli, C. Alippi, F. Grimaccia N. Haritos, and R. E. Zich, "Smart Environmental Measurement \& Analysis Technologies (SEMAT): Wireless sensor networks in the marine environment". In Proceedings of Wireless Sensor and Actuator Network Research on Opposite Sides of the Globe (SENSEI), 2008.

[5] T. S. Myers, I. M. Atkinson, and R. Johnstone, "Supporting coral reef ecosystems research through modelling a re-usable ontology framework", Applications of Artificial Intelligence, 90(24), 2009.

[6] S. Tilak, P. Hubbard, M. Miller, and T. Fountain, "The Ring Buffer Network Bus (RBNB) Data Turbine Streaming Data Middleware for Environmental Observing Systems". In Proceedings of the third IEEE International Conference on eScience and Grid Computing, 2007.

[7] J. Trevathan, I. M. Atkinson, W. Read, R. Johnstone, N. Bajema and J. McGeachin, "Establishing Low Cost Aquatic Monitoring Networks for Developing Countries", In Proceedings of the International Conference on Wireless Communications and Information Technology in Developing Countries, pp. 37-48, 2010. 